\title{
Directed polymer in random media, in two dimensions : numerical study of the aging dynamics
}

\author{
A. Barrat* \\ Laboratoire de Physique Théorique de l'Ecole Normale Supérieure ${ }^{\dagger}, 24$ rue Lhomond, 75231 Paris \\ Cedex 05, France
}

\begin{abstract}
Following a recent work by Yoshino, we study the aging dynamics of a directed polymer in random media, in $1+1$ dimensions. Through temperature quench, and temperature cycling numerical experiments similar to the experiments on real spin glasses, we show that the observed behaviour is comparable to the one of a well known mean field spin glass model. The observation of various quantities (correlation function, "clonation" overlap function) leads to an analysis of the phase space landscape.
\end{abstract}

LPTENS preprint 96/57

Typeset using REVTEX

\footnotetext{
*Present address : International Center for Theoretical Physics, Strada Costiera 11, 34100 Trieste, Italy ; email barrat@ictp.trieste.it

†Unité propre du CNRS, associée à l'Ecole Normale Supérieure et à l'Université de Paris Sud
} 


\section{INTRODUCTION}

The study of directed polymers in random media has triggered a lot of interest and of works (for a recent review see [1]), since it is related to many fields, from the fluctuations of interfaces [2] to quantum mechanical problems in a time-dependent random potential [3], or the very topical problem of vortex lines in high temperature superconductors [4]. It has also connections with spin glasses, as has been shown by Derrida and Spohn [5], who studied a mean field version (on a Cayley tree) of the random polymer, showing the existence of a low temperature phase similar to the Random Energy Model of Derrida [6].

In finite dimensions $(d+1$, with $d$ transverse dimensions), the existence of a phase transition has been shown for $d \geq 2[0]$, whereas the system is always in a low temperature phase for $d=1$, and has been called a "baby spin glass" in [10].

The dynamics of such model has not been much studied so far. A recent numerical work by Yoshino [11] has made clear the existence of aging for the directed polymer in random media in dimensions $1+1$, with violation of time translation invariance and of the fluctuation-dissipation theorem. The observed behaviour for the correlation function is similar to the scaling properties for the simulations of a three dimensional spin glass model [12]. It is analyzed along the lines of a scenario similar to the droplets model for spin glasses [13,14] : the polymer moves in a network of "tubes" where its probability of presence (calculated at equilibrium with a transfer matrix method) is high. The network has a quite complicated spatial structure, and the tubes form loops of various sizes. The dynamics consists then of rapid fluctuations inside the tubes (acting as traps) combined with thermally activated jumps between different tubes. These thermal excitations are compared to the droplets excitations. Besides, the fact that the loops display a broad distribution of sizes [15] induces a broad distribution of relaxation times, and thus aging.

On the other hand, Cugliandolo, Kurchan and Le Doussal, following the study of the aging dynamics of a mean-field spin glass model [16], and of a particle in an infinite dimensional random potential [17, 18], have proposed an analytical treatment of the long times 
off equilibrium dynamics of an elastic manifold embedded in an infinite dimensional space, in the presence of a quenched random potential [19] (the statics of such a model has been studied by Mézard and Parisi with a replica variational Gaussian approximation, becoming exact in this infinite dimensional limit [20]). This corresponds here to an infinite $d$. The finite dimension of the manifold leads to the study of the relaxation of its Fourier modes $k$. The two-times correlation and response functions $C_{k}\left(t, t^{\prime}\right)$ and $r_{k}\left(t, t^{\prime}\right)$ satisfy dynamical equations where two regimes can be separated, as for the case of the particle [17,18]) : a stationary regime, and a regime displaying aging, where the properties of equilibrium dynamics (namely, time translation invariance and fluctuation dissipation theorem) are violated. Various equations can be written for the long times behaviour of the functions, for example, the measure of the violation of the fluctuation dissipation theorem $X(C)$ does not depend on $k$.

We will here focus on the same model as [11]; after describing this model, the used dynamics, we perform several numerical experiments for aging dynamics. Preparing the polymer in its ground state, we also show that it can display stationary dynamics, and compare the two kinds of dynamics in order to analyse the phase space landscape.

\section{A. Model}

The polymer is defined on a square lattice of linear size $N$ : it consists of $N$ monomers lying on the sites $\left\{\left(i, x_{i}\right), i=1, \cdots, N\right\}$, and the Hamiltonian is :

$$
H\left[\left\{x_{i}\right\}\right]=\sum_{i=2}^{N}\left(\left|x_{i}-x_{i-1}\right|+V\left(i, x_{i}\right)\right),
$$

where $V$ is a Gaussian random potential, with zero mean and variance $\sigma$, uncorrelated from site to site. The first term gives the elastic energy ; moreover, the steps $\left|x_{i}-x_{i-1}\right|$ are

restricted to 0 or 1 , and one of the extremities is fixed, so that a Boltzmann measure can be defined : $x_{1}=N / 2$.

Transfer matrix method can be used to study the statics of this model [10]. In particular, the Edwards-Anderson parameter can be calculated, following [10]: we take two replicas 
of the polymer, with the same realization of the disordered potential ; if $Z(x, y, L)$ is the partition function for the pairs of polymers arriving at transverse coordinates $x$ and $y$ after $L$ steps (or monomers), and if $\tilde{Z}(x, y, q, L)$ is the partition function restricted to such pairs having an overlap $q$, it is possible to write recursion relations in $L$ for $Z$ and $Y(x, y, L)=$ $\sum_{q} q \tilde{Z}(x, y, q, L)$, and therefore to evaluate

$$
\lim _{L \rightarrow \infty} \frac{1}{L} \frac{\sum_{x, y} Y(x, y, L)}{\sum_{x, y} Z(x, y, L)},
$$

and to average it over disorder to obtain $q_{E A}$. We will use this value to check some expected long time limits of dynamical quantities (see below).

The directed polymer is evolving with Monte-Carlo dynamics in a heat bath in the following way : a monomer and a move are chosen at random, and the move is performed with probability $\min (1, \exp (-\beta \Delta E))$ (Metropolis algorithm), $\beta$ being the inverse temperature, and $\Delta E$ the change of energy involved. One Monte-Carlo step consists in $N$ such tries.

\section{B. Numerical experiments}

The transfer matrix method allows us to find the ground state in a given realization of a potential ; we have studied the dynamics in two cases : the initial configuration is either taken at random, or as the ground state. The polymer, therefore coming from an infinite temperature or from a zero temperature thermalized state, is then free to evolve at the temperature of the heat bath. We then measure the evolution of the energy, the two-times correlation function defined by

$$
C\left(t_{w}+t, t_{w}\right)=\overline{\left\langle\sum_{i=1}^{N} \delta_{x_{i}\left(t_{w}+t\right), x_{i}\left(t_{w}\right)}\right\rangle}
$$

where $\langle$.$\rangle is a mean over thermal noise and the overline denotes a mean over realizations of$ the quenched disorder, and the overlap between two copies of the polymer evolving in the same realization of the potential, with the same thermal noise until $t_{w}$, and then decoupled

[21-24] (a process called "clonation" in [21]); if these copies are labeled by ${ }^{(1)}$ et ${ }^{(2)}$, this overlap is 


$$
Q_{t_{w}}\left(t_{w}+t, t_{w}+t\right)=\overline{\left\langle\sum_{i=1}^{N} \delta_{x_{i}^{(1)}\left(t_{w}+t\right), x_{i}^{(2)}\left(t_{w}+t\right)}\right\rangle} .
$$

Besides, we have performed temperature cycling experiments in the same way as for real spin glasses [25]. Most of the runs have been made with a polymer of length $N=500$, and some with $N=800$ : no finite size effects were seen for the used simulation times.

\section{AGING DYNAMICS}

\section{A. Quench at initial time}

The initial configuration of the polymer is chosen at random, with the constraint $\mid x_{i}-$ $x_{i-1} \mid=0$ or 1 ; then it evolves at a fixed temperature. It has already been observed [11] that the correlation function $C\left(t_{w}+t, t_{w}\right)$ previously defined displays aging behaviour : it depends explicitly on $t_{w}$ and $t$ (see figure (1)) ; as the system ages, it becomes more rigid, in the sense that it evolves slower and gets away from himself always slower.

For times $t$ much lower than $t_{w}$, the dynamics has the characteristics of equilibrium dynamics : $C\left(t_{w}+t, t_{w}\right)$ depends only on $t$ (time translation invariance), and it has been checked numerically [11] that the fluctuation-dissipation theorem is valid. Besides, we have also checked the validity of the relation $Q_{t_{w}}\left(t_{w}+t, t_{w}+t\right)=C\left(t_{w}+2 t, t_{w}\right)$ [24]. In this regime, we have therefore a quasi-equilibrium dynamics. Some well-known spin glass models (like for example the $p$-spin spherical model [16], or the random manifold [17,19]) present a correlation function decaying from 1 to $q_{E A}$, with a power law approach to the $q_{E A}$ plateau : $q_{E A}+A t^{-\nu}$

We have been able to fit the results of the simulations with such form, using the values of $q_{E A}$ obtained by transfer matrix method : this fits therefore use only 2 parameters, and not 3 (see figure (3) for the values, and figure (11) for examples of the fits). The obtained values of $\nu$ (typically in the range $0.1-0.2$ ) are displayed in figure (2).

For times $t$ comparable or bigger than $t_{w}$, the correlation function $C\left(t_{w}+t, t_{w}\right)$ depends explicitly on $t_{w}$ : the dynamics is no more time translation invariant. For $t \gg t_{w}$, it decays 
as a power law,

$$
C\left(t_{w}+t, t_{w}\right) \simeq f\left(t_{w}, T\right)\left(t / t_{w}\right)^{-\lambda}
$$

We are therefore in presence of a weak-ergodicity breaking behaviour, $\lim _{t \rightarrow \infty} C\left(t_{w}+t, t_{w}\right)=$ 0 [26]. The obtained values of $\lambda$ are comparable to the values of $\nu$ (see figure (2)), and both exponents are increasing functions of temperature, like in real spin glasses [25].

It is important to note that the $\nu$ exponent is different from the $x$ exponent studied by Yoshino [11] : this $x$ is obtained by the scaling form

$$
C\left(t_{w}+t, t_{w}\right) \simeq t^{-x}
$$

for the $t \ll t_{w}$ part, with a global form

$$
C\left(t_{w}+t, t_{w}\right)=t^{-x} \Phi\left(t / t_{w}\right)
$$

If we take the limit $\lim _{t_{w} \rightarrow \infty} \lim _{t \rightarrow \infty}$ we obtain the same behaviour, but the opposite order of limits, $\lim _{t \rightarrow \infty} \lim _{t_{w} \rightarrow \infty} C\left(t_{w}+t, t_{w}\right)$ yields 0 , in contradiction with the expected static limit

$$
\lim _{t \rightarrow \infty} \lim _{t_{w} \rightarrow \infty} C\left(t_{w}+t, t_{w}\right)=q_{E A}
$$

which is also obtained by the form $q_{E A}+A t^{-\nu}$.

With numerical data it is however difficult to prefer one of these forms, and much longer simulations (much bigger values of $t_{w}$ ) would be necessary.

If we now look at the overlap between two replicas separated at $t_{w}, Q_{t_{w}}$, it seems that this function has a finite limit at large times $t$ (see figure (田), with a value compatible with the value of $q_{E A}$, for big enough $t_{w}$. This constatation puts this model in the class I of the classification of [24], which includes domain-growth models, and the $p=2$ spherical $p$-spin model [23]: it indicates that the evolution in phase space takes place in "corridors" [21], of size $q_{E A}$. On the contrary, the case of the manifold embedded in a infinite dimensional space yields $\lim _{t_{w} \rightarrow \infty} \lim _{t \rightarrow \infty} Q_{t_{w}}\left(t_{w}+t, t_{w}+t\right)=0$, and therefore belongs to type II, which probably indicates a much more complex phase-space landscape, and occurs also for example for the $p$-spin spherical model with $p \geq 3$ [24]. 


\section{B. Temperature cycling experiments}

A spin-glass quenched under its transition temperature, and then submitted to temperature changes, shows a very puzzling behaviour (see [25] for a review, and references therein) that we briefly describe now. After a quench at time $t=0$, the temperature cycle is as follows (see figure (5)) : the temperature is $T$ from $t=0$ to $t=t_{1}$, then $T+\Delta T$ from $t=t_{1}$ to $t=t_{2}$, and again $T$ after $t=t_{2} ; \Delta T$ can be negative as well as positive. For a positive $\Delta T$, one observes a reinitialization of the dynamics, with, e.g. for a thermoremanent magnetization, a relaxation after $t_{2}$ identical to the one obtained after a quench at $t=0$ and a waiting time $t_{e f f}=t_{w}-t_{2}$. On the contrary, for a negative $\Delta T$, the system keeps memory of its evolution, and its relaxation corresponds to an effective age between $t_{w}$ and $t_{w}-t_{2}+t_{1}$

We have performed these jumps numerically with $T=2, \Delta T=1,2,-1$, and $t_{1}=500$, $t_{2}=1500$. We have then monitored the evolution of the energy of the polymer, as well as $C_{\text {jump }}\left(t_{w}+t, t_{w}\right)$ with $t_{w}=2000$, comparing these quantities with the ones obtained for $\Delta T=0$ (constant temperature).

It is clear (figure (6)) that $C_{\text {jump }}\left(t_{w}+t, t_{w}\right)$ corresponds to a certain $C\left(t_{\text {eff }}+t, t_{\text {eff }}\right), t_{\text {eff }}$ being an effective age for the system, depending on $t_{2}-t_{1}$ and on $\Delta T ; t_{\text {eff }}$ is less than $t_{w}$ for a negative $\Delta T$, which means that the dynamic has been slowed down by the time spent at a lower temperature ; here we estimate $t_{\text {eff }}=1000=t_{w}-t_{2}+t_{1}$ (for $\Delta T=-1$, the data corresponding to $C_{\text {jump }}(2000+t, 2000)$ with $t_{1}=500, t_{2}=1500$ are superimposed on the curve $C(1000+t, 1000))$. For smaller values of $\Delta T$, or longer times $t_{2}-t_{1}, t_{e f f}$ can be bigger than $t_{w}-t_{2}+t_{1}$ : the time spent at $T+\Delta T$ can contribute a little to the aging. For positive values of $\Delta T, t_{e f f}$ is bigger than $t_{w}$ (in figure (6), $t_{e f f}=3000$ ), showing that the time spent at $T+\Delta T$ has contributed to the approach to equilibrium more than the same time at $T$.

The behaviour of the directed polymer is therefore symmetrical for positive or negative variations of temperature. No reinitialization of dynamics is found. This type of behaviour 
is similar to the one observed for mean-field spin glasses [25,23], and thus very different from the one observed in real experiments on spin glasses.

It should be remarked that real experiments deal with response function, whereas we are monitoring correlation functions. However, numerical simulations of a three dimensional Ising spin glass model have shown also for the correlation functions [27] a partial reinitialization of the dynamics for positive $\Delta T$, and asymmetric outcomes of numerical experiments with positive or negative temperature cycles. Nothing of this kind is found here.

\section{RELAXATION FROM THE GROUND STATE}

The transfer matrix method allows to find the ground state of the polymer, given a realization of the potential. We then let the polymer evolve at temperature $T$ as before.

The measure of the correlation function $C\left(t_{w}+t, t_{w}\right)$ for various $t_{w}$ and $t$ shows in this case a simple $t$ dependence : the system is time translation invariant (figure (7). Besides, $C\left(t_{w}+t, t_{w}\right)$ does not seem to go to zero for large $t$, or at least stays well above the correlation at same times, for a system with random initial condition, for the accessible times : no power law decay $t^{-\lambda}$ is found. It seems that ergodicity is really, and not simply weakly, broken.

We have also studied the dynamics for an initial configuration of energy close to the ground state energy, but spatially well separated. In this case, we observe a similar behaviour, with a time translation invariant correlation function.

Such behaviour has been observed for example in the TAP states of the $p$-spin spherical model with $p \geq 3$ [28].

\section{ENERGY}

We have monitored the evolution of the energy density $E_{a l}$ of the polymer ; for a random initial condition the initial energy is high, so the initial behaviour is a fast decay, followed by a much slower evolution. 
When the polymer is prepared in its ground state on the contrary, its energy $E_{f}$ grows quickly because of the thermal bath, and then stays constant.

We show in figure (8) the difference between the energy densities for both situations, in logarithmic scales : this plot shows that the evolution is compatible with a power law decay of $E_{a l}$ towards $E_{f}$. All the aging dynamics take therefore place at higher energy densities than those of the low lying states, where the dynamics is stationary.

\section{DISCUSSION}

Whereas the previous analysis of the dynamics made by Yoshino was based on an analogy with the droplets model [13,14], we focus here more on a phase space analysis.

It must however be clear that, as for many numerical simulations, especially for glassy systems, the available time scales remain quite small, and that the results should therefore be considered as tendencies, indications of behaviour. They allow us, with these precautions, to present the following analysis.

The observed behaviour is quite similar to the one found for the spherical $p$-spin model with $p=2$ : the dynamic consists in a slow search of the ground state, with a slowly decaying energy. There exists many states with low energy, but the polymer is not able to find them, during its aging dynamics : it remains at higher energy density ; on the contrary, if it is put in one of these configurations, it stays trapped and has a stationary dynamics.

The behaviour of the overlap of two copies of the system, having same configurations until a certain time, and then decoupled, shows besides that the evolution takes place in some kind of "gutters" in phase space. The results of temperature cycling experiments also show that the directed polymer in $1+1$ dimensions is a much simpler system than real spin glasses. These two results are probably related, and also in agreement, concerning the relative simplicity of the phase space, with the fact [11] that the response to a tilt field applied at the end of the polymer does not display any aging ; however it would be nice to measure the response to a spatially sinusoidal field, and the relaxation after cutting the 
field, to check if such a relaxation has aging, and how it depends from the wavelength of the field. However, the sample to sample fluctuations of the response functions are very important, and such measurements are therefore very difficult.

These behaviours are in fact intermediate between the $p=2$ spherical $p$-spin model (analogous to domain growth [23) and the $p \geq 3$ case which, despite being a mean field model, displays a much more complicated behaviour with long term memory and a very complex phase space [16].

It would certainly be very interesting to study the directed polymer dynamics in higher dimensions : these new dimensions could provide a way to avoid energy barriers by going around them. The appearance of these entropy barriers [29 32] could yield new interesting effects and a richer dynamic in a more complex phase space.

It is a pleasure to thank M. Mézard who initiated this work and R. Monasson for useful discussions and comments.

\section{REFERENCES}




\section{REFERENCES}

[1] T. Halpin-Healy and Y.-C. Zhang, Phys. Rep. 254, 215 (1995).

[2] D. A. Huse and C. L. Henley, Phys. Rev. Lett. 54, 2708 (1985).

[3] M. Kardar, Nucl. Phys. B 290, 582 (1987).

[4] G. Blatter, M. V. Feigel'man, V. B. Geshkenbein, A. I. Larkin, and V. M. Vinokur, Rev. Mod. Phys. 66, 1125 (1994).

[5] B. Derrida and H. Spohn, J. Stat. Phys. 51, 817 (1988).

[6] B. Derrida, Phys. Rev. Lett. 45, 79 (1980).

[7] J. Z. Imbrie and T. Spencer, J. Stat. Phys. 52, 609 (1988).

[8] J. Cook and B. Derrida, J. Stat. Phys. 57, 89 (1989).

[9] B. Derrida and O. Golinelli, Phys. Rev. A 41, 4160 (1990).

[10] M. Mézard, J. Phys. France 51, 1831 (1990).

[11] H. Yoshino, J. Phys. A 29, 1421 (1996).

[12] H. Rieger, Annual Reviews of Computational Physics II, 295 (1995).

[13] D. S. Fisher and D. A. Huse, Phys. Rev. Lett. 56, 1601 (1986).

[14] D. S. Fisher and D. A. Huse, Phys. Rev. B 38, 373 (1988).

[15] T. Hwa and D. S. Fisher, Phys. Rev. B 49, 3136 (1994).

[16] L. F. Cugliandolo and J. Kurchan, Phys. Rev. Lett. 71, 173 (1993).

[17] S. Franz and M. Mézard, Physica A 210, 48 (1994).

[18] L. F. Cugliandolo and P. L. Doussal, Phys. Rev. E 53, 1525 (1996).

[19] L. F. Cugliandolo, J. Kurchan, and P. L. Doussal, Phys. Rev. Lett. 76, 2390 (1996). 
[20] M. Mézard and G. Parisi, J. Physique I 1, 809 (1991).

[21] A. Baldassarri, Non-equilibrium Monte Carlo dynamics of the Sherrington Kirkpatrick mean field spin glass, condmat 9607162 (1996).

[22] A. Baldassarri, Studia della dinamica fuori dall'equilibrio di un modello di vetro di spin in campo medio, Master's thesis, Universitá degli studi di Roma "La Sapienza", 1995.

[23] L. F. Cugliandolo and D. S. Dean, J. Phys. A 28, 4213 (1995).

[24] A. Barrat, R. Burioni, and M. Mézard, J. Phys. A 29, 1311 (1996).

[25] E. Vincent, J. Hammann, M. Ocio, J. P. Bouchaud, and L. F. Cugliandolo, Slow dynamics and aging in spin glasses, preprint condmat 9607224 (1996).

[26] J. P. Bouchaud, J. Phys. I France 2, 1705 (1992).

[27] H. Rieger J. Phys. I France 4, 883 (1994).

[28] A. Barrat, R. Burioni, and M. Mézard, J. Phys. A 29, L81 (1996).

[29] A. Barrat and M. Mézard, J. Phys. I (France) 5, 941 (1995).

[30] F. Ritort, Phys. Rev. Lett. 75, 1190 (1995).

[31] S. Franz and F. Ritort, Europhys. Lett. 31, 507 (1996).

[32] S. Franz and F. Ritort, condmat 9508133, to appear in J. Stat. Phys. (1996). 


\section{FIGURES}
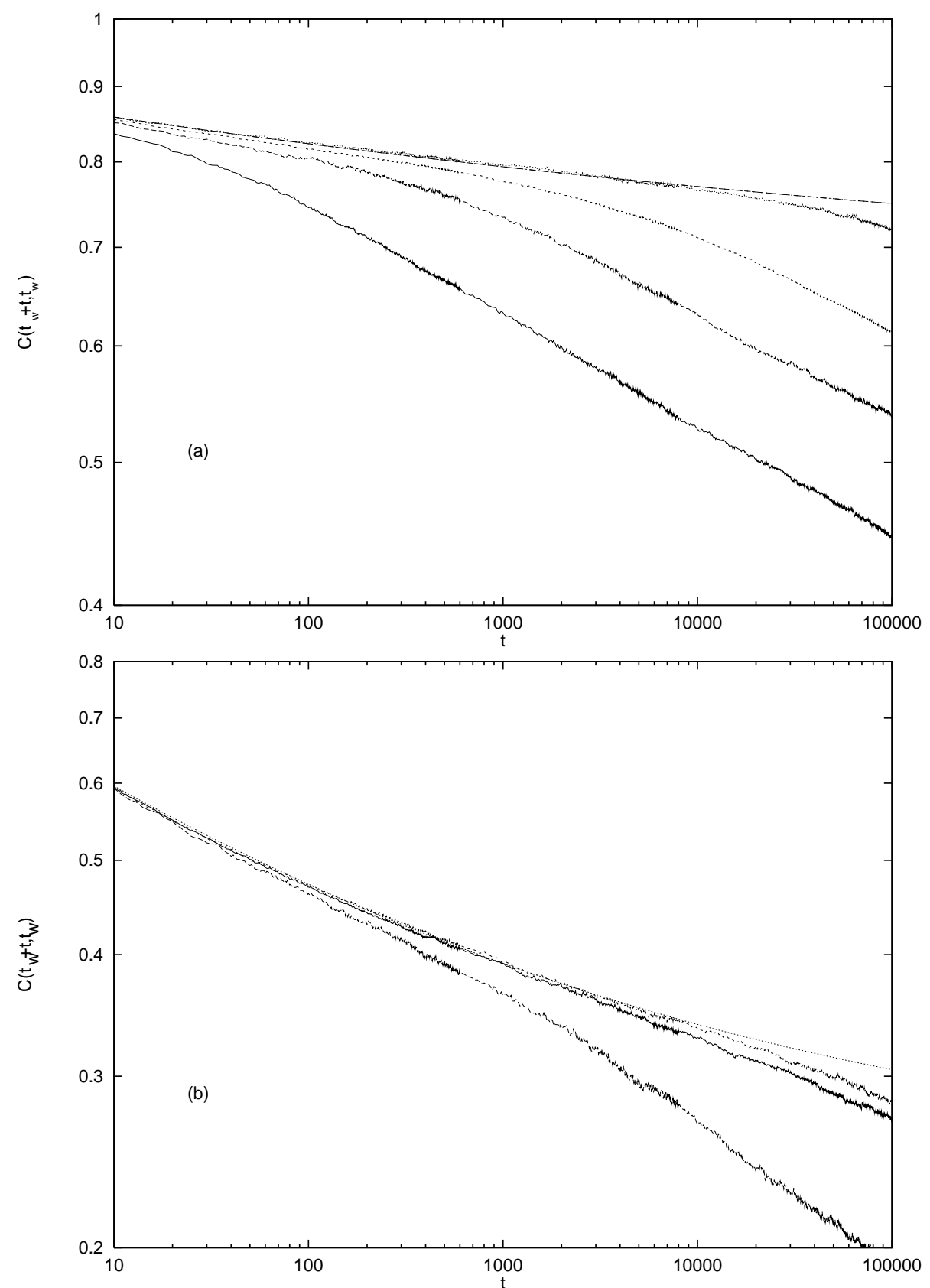

FIG. 1. (a) : from bottom to top, $C\left(t_{w}+t, t_{w}\right)$ versus $t$ for $t_{w}=50,500,5000,100000$, at $T=1$, and the fit $C_{a s}(t)=0.65+.25 t^{-0.08}$. (b) : $C\left(t_{w}+t, t_{w}\right)$ versus $t$ for $T=3$, and $t_{w}=2000,50000,100000$, and $C_{a s}(t)=0.24+0.545 t^{-0.185}$. In both cases, $q_{E A}(T=1)=0.65$ and $q_{E A}(T=3)=0.24$ have been obtained by transfer matrix method. 


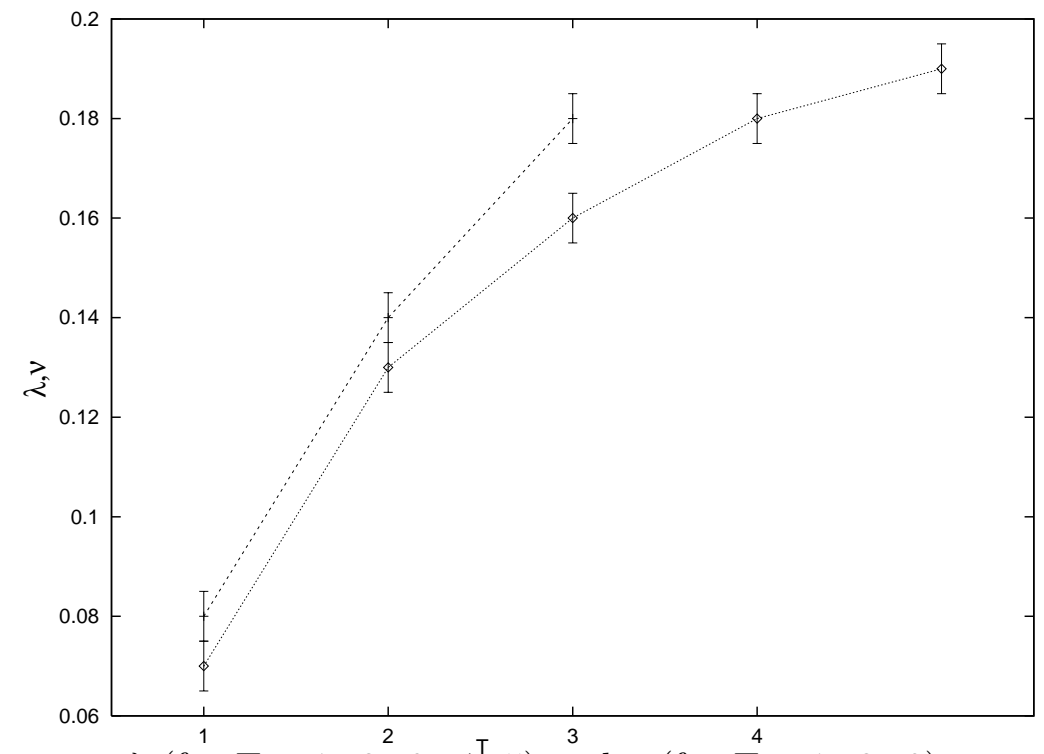

FIG. 2. Exponents $\lambda$ (for $T=1,2,3,4,5$ ) and $\nu$ (for $T^{\top}=1,2,3$ ) versus temperature.

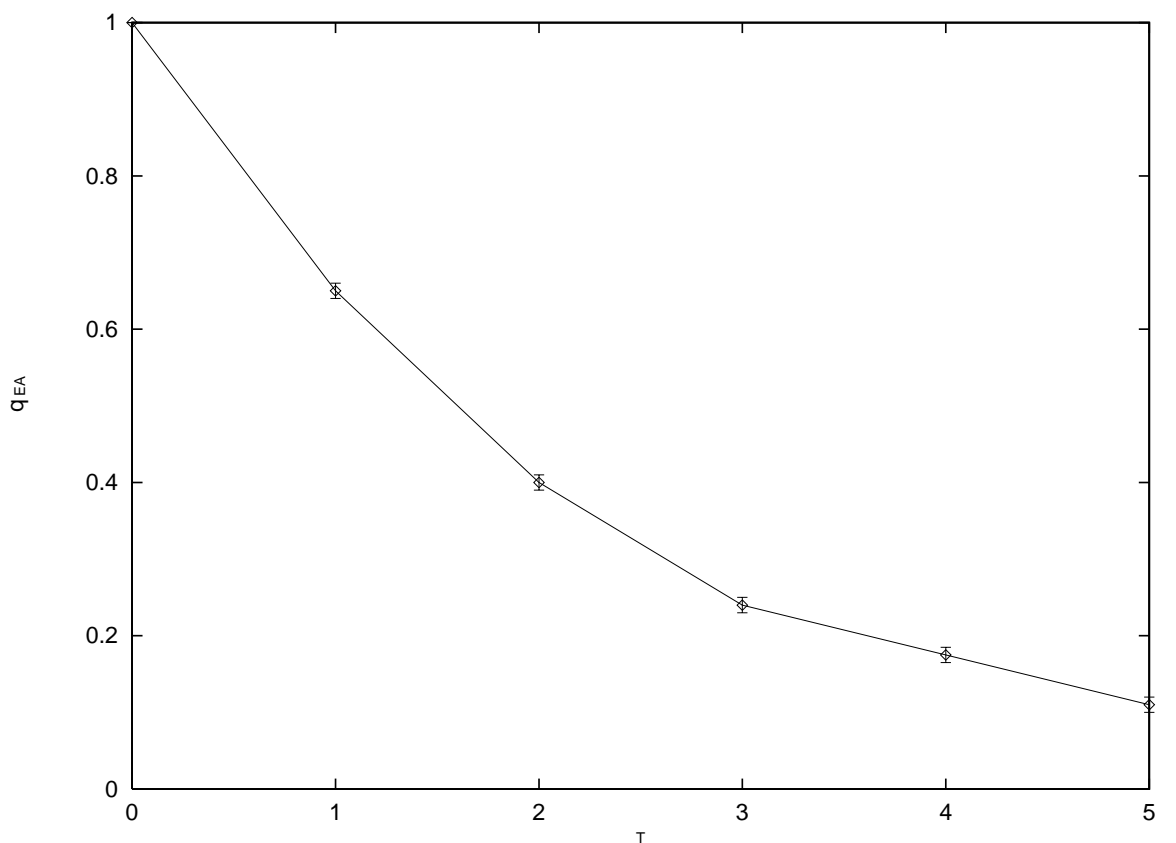

FIG. 3. Values of $q_{E A}$ obtained by transfer matrix method, versus temperature. 

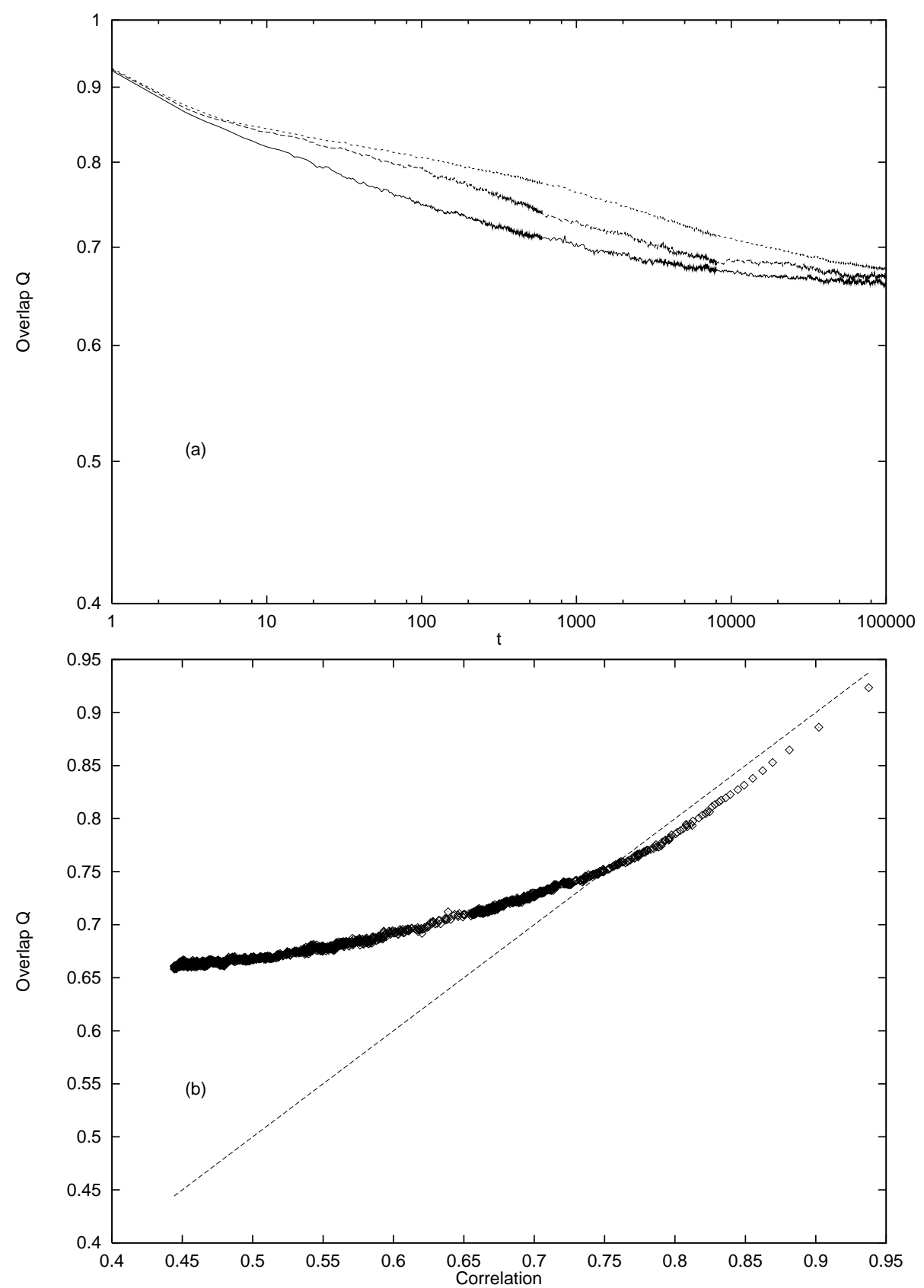

FIG. 4. $Q_{t_{w}}\left(t_{w}+t, t_{w}+t\right)$ for $t_{w}=50,500,5000, T=1$, in logarithmic scale (a) ; $Q_{t_{w}}\left(t_{w}+t, t_{w}+t\right)$ versus $C\left(t_{w}+t, t_{w}\right)$ for $t_{w}=50, T=1(\mathrm{~b})$. At $T=1, q_{E A} \approx 0.65$. 


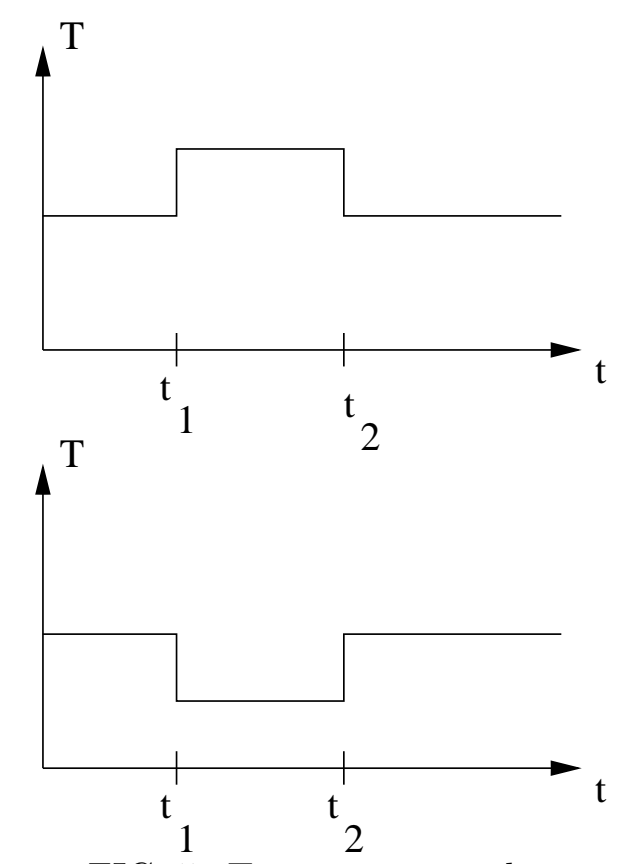

FIG. 5. Temperature cycles.

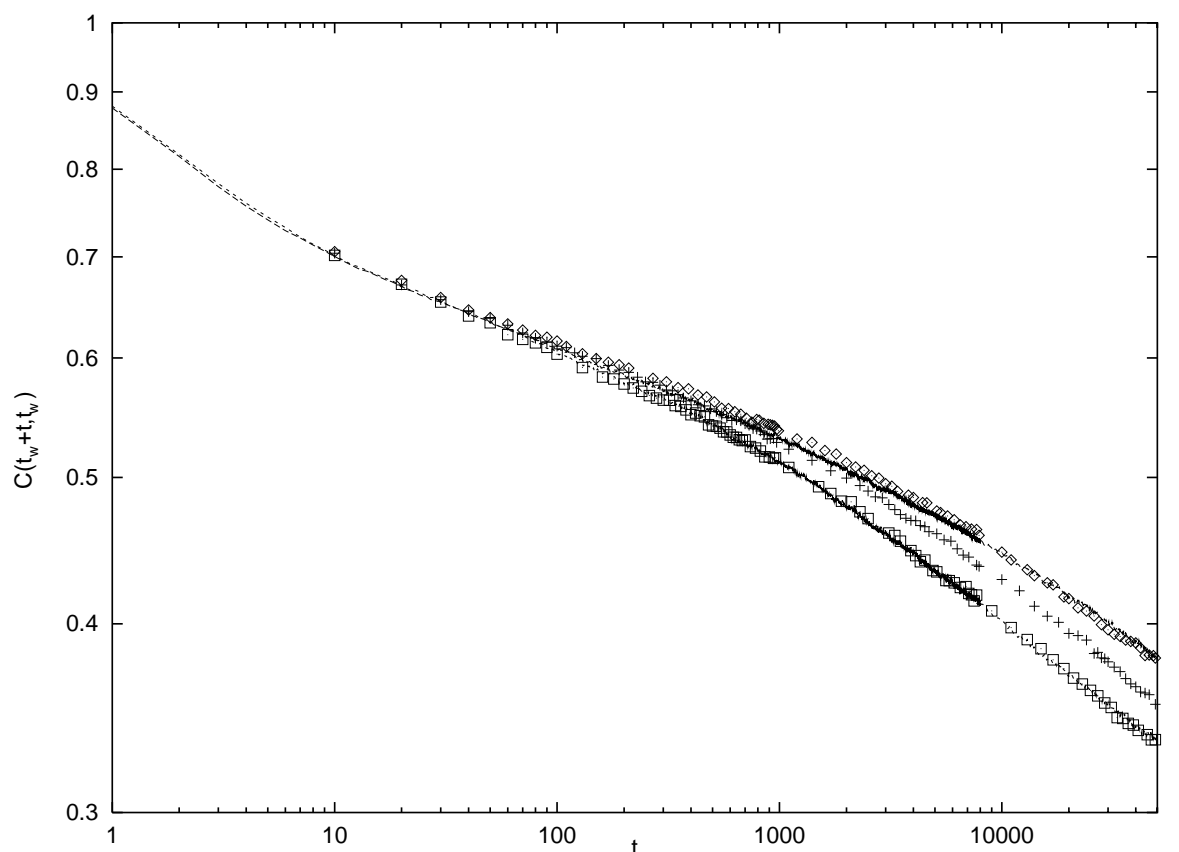

FIG. 6. Results of the temperature cycling experiments : The symbols show $C\left(t_{w}+t, t_{w}\right)$ at constant temperature $T=2$ for $t_{w}=1000$ (squares), $t_{w}=2000$ (crosses), $t_{w}=3000$ (diamonds). The temperature cycles are done with $t_{1}=500, t_{2}=1500, t_{w}=2000, T=2$, and yield $C_{\text {jump }}\left(t_{w}+t, t_{w}\right):$ the corresponding curves are superimposed on $C(1000+t, 1000)$ for $\Delta T=-1$, and on $C(3000+t, 3000)$ for $\Delta T=1$. 

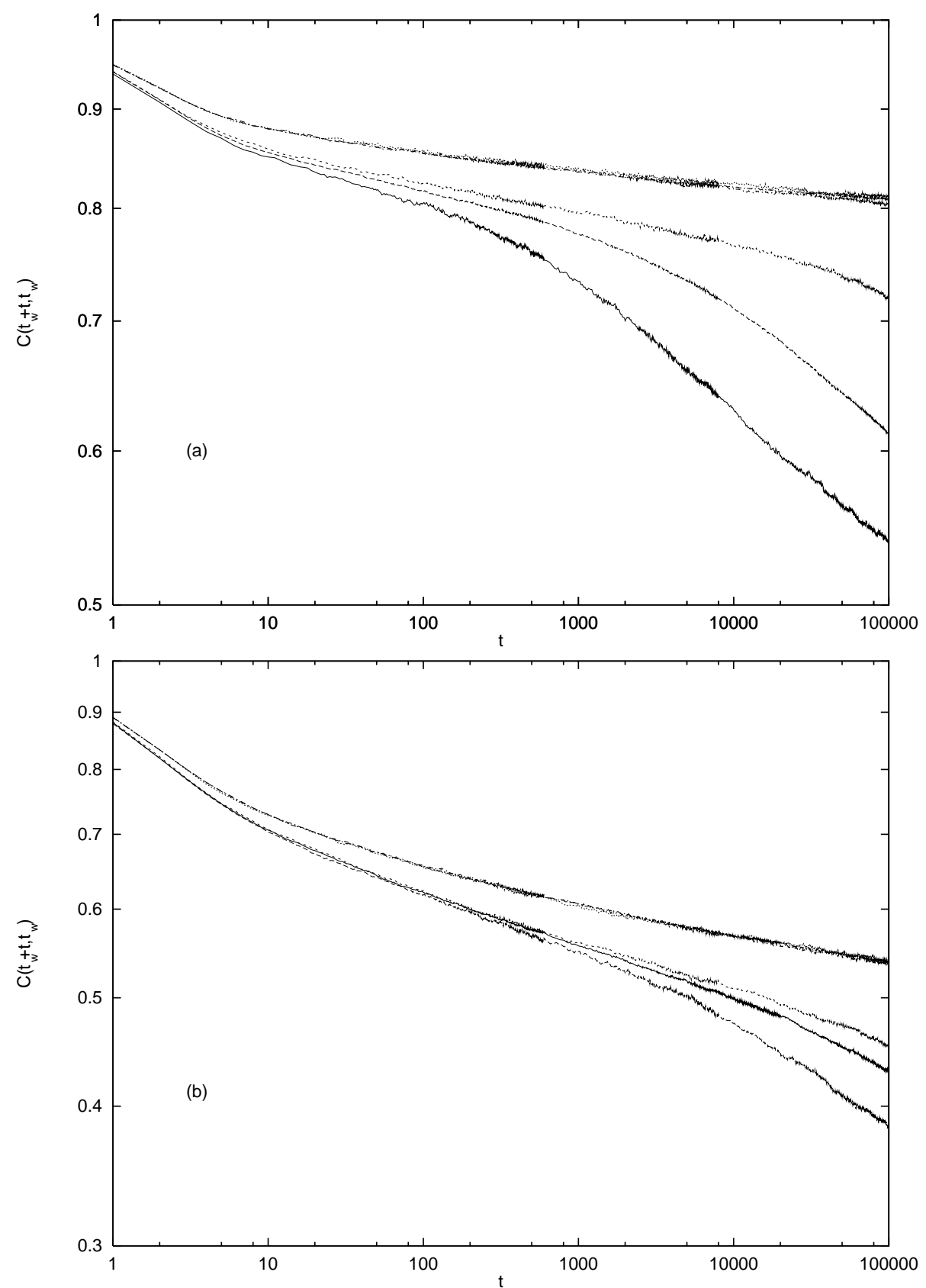

FIG. 7. $C\left(t_{w}+t, t_{w}\right)$ versus $t$, in logarithmic scales : comparison of the evolutions for various initial conditions. For each figure, the three lower curves correspond to random initial conditions ((a), for $T=1, t_{w}=500,5000,100000$, and (b), for $\left.T=2, t_{w}=10000,50000,100000\right)$, while three curves corresponding to the system being prepared in its ground state at initial time are superimposed onto each other (with the same waiting times $t_{w}$ as for the lower curves), showing that $C\left(t_{w}+t, t_{w}\right)$ depends only on $t$ in this case. 

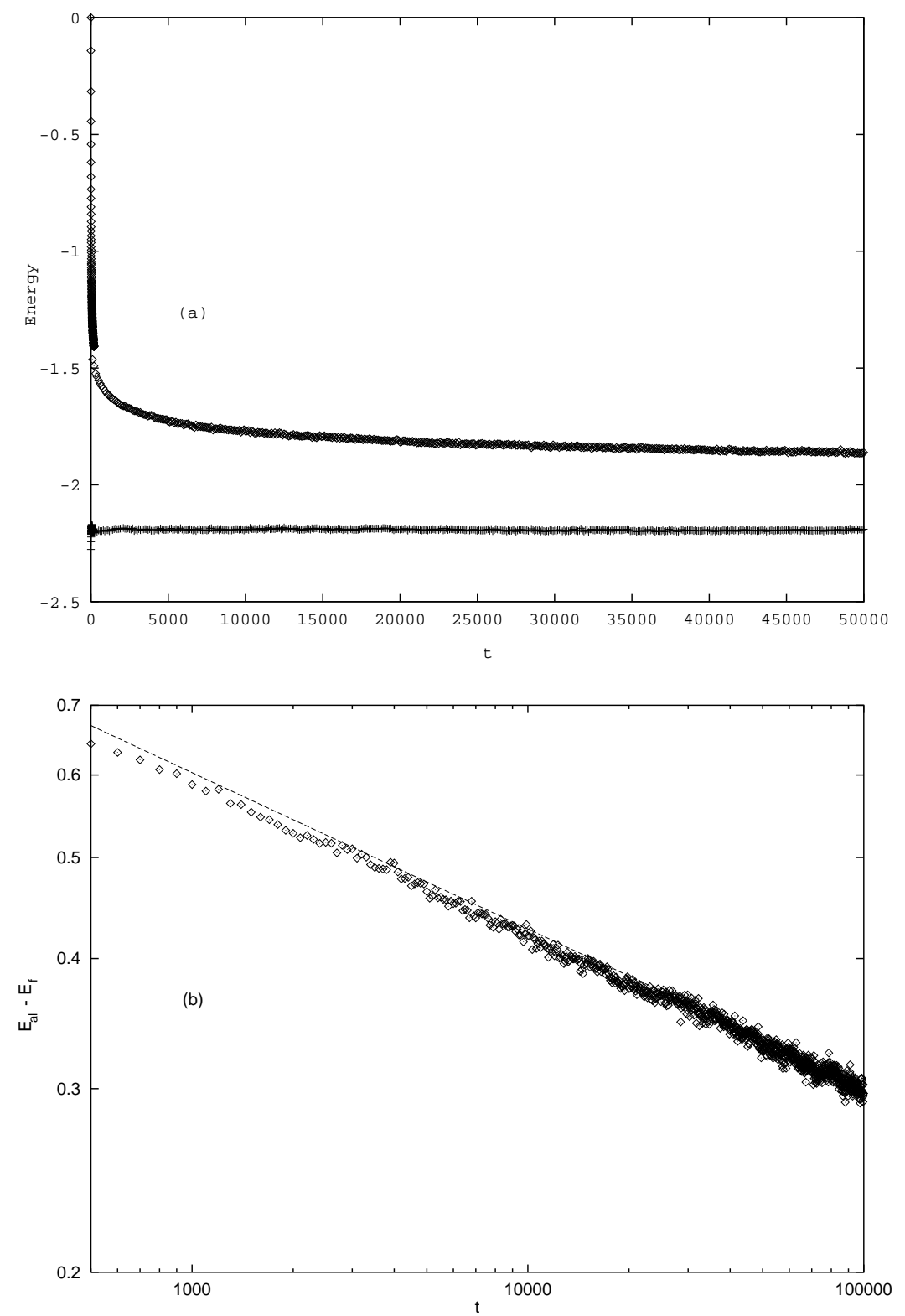

FIG. 8. (a) : evolution of the energies as a function of time, for $T=2$, for random initial conditions (upper curve), or for a system prepared in its ground state (lower curve). (b) : $E_{a l}-E_{f}$ versus time, for $T=2$, in logarithmic scale (symbols) ; the straight line corresponds to the power law $t^{-.15}$. 